\title{
FUNCTIONALIZED CYCLODEXTRINS FOR TARGETED DRUG TRANSPORT
}

\section{M.V. Papezhuk, A.V. Chemodanova, V.A. Volynkin, V.T. Panyushkin}

Kuban State University, 350040, Russia, Krasnodar,st. Stavropolskaya, 149.

DOI: 10.19163/MedChemRussia2021-2021-502

E-mail: marina-marina322@mail.ru

CDs are cyclic oligosaccharides composed of varying numbers of glucopyranose units. CDs have a characteristic truncated cone-shaped structure with a cavity of different sizes which depends on the type. The outer surface of the $C D$ is hydrophilic due to the presence of hydroxyl groups and the inner surface is hydrophobic because lined with $\mathrm{H} 3$ and $\mathrm{H} 5$ atoms. Due to their unique structure these compounds are capable of forming guest-host-type inclusion complexes with organic, inorganic and organometallic molecules. This phenomenon makes the use of $C D$ in the pharmaceutical industry attractive. they are able to perform not only transport the function and the active substance storage, but also allow changing the physical properties of the active components in the desired direction.

In its native form $\beta-C D$ is not an ideal drug carrier due to its low solubility. The functionalization of $\beta-C D$ makes it possible to significantly increase the solubility of compounds, the derivative itself can also act as a biologically active substance and functional groups can serve as an additional coordination center with metal ions. $\beta-C D$ is relatively easily amenable to chemical modification at the primary hydroxyl groups at $C_{6}$, then at the secondary hydroxyl groups at $\mathrm{C}_{2}, \mathrm{C}_{3}$.

A number of $\beta-C D$ derivatives have been obtained. Carboxymethyl- $\beta-C D$ was received directly by the interaction of chloroacetic acid with $\beta-C D$ in a strongly alkaline medium. Mono-6-0-benzoyl- $\beta$-cyclodextrin was synthesized in order to develop the esterification technique as applied to CD. An interesting feature is that the reaction proceeds over primary hydroxyl groups with monosubstitution. Mono-6-0-nicotinoyl- $\beta-C D$ was synthesized by the esterification reaction. The tosyl derivative was obtained as an intermediate, because tosylate can be easily displaced by other nucleophiles, e.g. 2-mercaptonicotinic acid. The successful preparation of the compounds has been proven by IR and NMR spectroscopy. 